\title{
Alterations of Cardiac $\mathbf{K}_{\mathrm{ATP}}$ Channels and Autophagy Contribute in the Late Cardioprotective Phase of Exercise Preconditioning
}

\author{
Jiao Lu, ${ }^{1} \mathrm{MD}$, Shan-Shan Pan, ${ }^{1} \mathrm{PhD}$, Qing-Tang Wang, ${ }^{1}$ MD and Yang Yuan, ${ }^{1} \mathrm{MD}$
}

\begin{abstract}
Summary
The cardiac effects of exercise preconditioning (EP) are well established; however, the mechanisms involving cardiac ATP-sensitive potassium channel $\left(K_{\text {ATP }}\right.$ channel) subunits and autophagy are yet to be fully established. The present work aims to investigate the alterations of cardiac $K_{\text {ATP }}$ channel subunits Kir6.2, SUR2A, and autophagy-related LC3 during the late cardioprotective phase of EP against exhaustive exercise-induced myocardial injury. Rats run on treadmill for four running time intervals, each with 10 minutes running and rest. Exhaustive exercise was performed $24 \mathrm{~h}$ after EP. Cardiac biomarkers, cTnI and NT-proBNP, along with the histological stain, were served as indicators of myocardial injury. Cardiac $\mathrm{K}_{\text {ATP }}$ channel subunits Kir6.2 and SUR2A were analyzed in this study, and autophagy was evaluated by LC3. The results revealed that EP reduced the exhaustive exercise-induced high level of serum cTnI and myocardial ischemia/hypoxia; however, it did not reveal any changes in the serum NT-proBNP level or cardiac BNP. Cardiac SUR2A mRNA significantly upregulated during the exhaustive exercise. The high levels of Kir6.2, SUR2A, LC3IIpuncta and LC3II turnover observed after exhaustive exercise were significantly mitigated by EP in the late phase. These results suggest that EP alleviates myocardial injury induced by exhaustive exercise through the downregulation of cardiac $\mathrm{K}_{\text {AтP }}$ channels and autophagy.
\end{abstract}

(Int Heart J 2018; 59: 1106-1115)

Key words: ATP-sensitive potassium channel, Cardioprotection, LC3, Exhaustive exercise, Myocardial injury

A single bout of exercise can enhance the tolerance of an ischemic heart to subsequent ischemic insult. This effect is known as exercise preconditioning (EP). ${ }^{1,2)} \mathrm{EP}$ has been found to play an important role in the reduction of myocardial infarction after subsequent ischemia reperfusion (I/R) injury, ${ }^{3)}$ prevention of myocardial stunning, ${ }^{4)}$ and even attenuation of isoproterenol-induced myocardial injury. ${ }^{5)} \mathrm{EP}$ contains two chronologically distinct phases: a short-lived early phase and a long-lived late phase. In previous studies, we have already revealed that EP can lead to cardioprotective effects regarding the reduction of exhaustive exerciseinduced myocardial injury, which might involve the translocation and activation of protein kinase $\mathrm{C}(\mathrm{PKC})$ in cardiomyocytes; ${ }^{1)}$ however, the mechanism of EP-induced cardioprotective effects is yet to be established.

\section{Editorial p.917}

The cardiac ATP-sensitive potassium channel ( $\mathrm{K}_{\text {ATP }}$ channel) comprised pore-forming Kir6.2 and regulatory SUR2A subunits. As a vital effector of EP intracellular signal transduction pathway, $\mathrm{K}_{\mathrm{ATP}}$ channel contribute to the preconditioning effect on infarct size lessen. ${ }^{2,6)}$ These stud- ies have investigated the preconditioning effect of exercise and participation of $\mathrm{K}_{\mathrm{ATP}}$ channel; however, alterations in the $K_{\text {ATP }}$ channel subunits during the cardioprotection of EP have rarely been reported. Although a study reported that exercise-mediated reductions in infarct size may involve the upregulation of cardiac sarcolemmal KATP channels, ${ }^{7)}$ researchers are yet to evaluate the changes in the $\mathrm{K}_{\text {ATP }}$ channel subunits in the EP-initiated cardioprotection against exhaustive exercise. Furthermore, considering that the EP-induced late cardioprotective effect is a complicated process concerning the maintenance of normal intracellular homeostasis, and that autophagy plays an essential role in mediating the stress-induced adaptation and damages in cells with limited capacity for regeneration and differentiation, we hypothesized that autophagy is involved in EP-induced late cardioprotection. Additionally, recent reports have revealed that autophagy increased during strenuous exercise to deal with exercise-induced metabolic disorders and peroxidation injury; ${ }^{8,9)}$ however, the role of autophagy in EP-induced late cardioprotective effects is poorly reported. Thus, the purpose of the present study was to demonstrate alterations in the transcriptional and translational levels of $\mathrm{K}_{\text {ATP }}$ channel subunits and preliminary discuss the role of autophagy in late cardiopro-

From the ${ }^{1}$ School of Kinesiology, Shanghai University of Sport, Shanghai, China.

This work is supported by the NSFC (National Natural Science Foundation of China) [grant number 31471136, 31071031]

Address for correspondence: Shan-Shan Pan, PhD, School of Kinesiology, Shanghai University of Sport, 399 Changhai Road, Shanghai 200438, China.

E-mail: panshanshan2013@163.com

Received for publication January 2, 2017. Revised and accepted May 16, 2017.

Released in advance online on J-STAGE August 11, 2018.

doi: 10.1536/ihj.17-003

All rights reserved by the International Heart Journal Association. 
Group

C

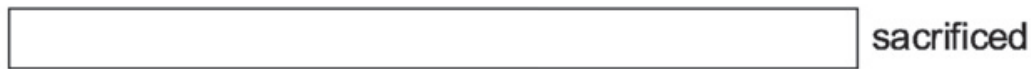

EE

exercise to exhaustion

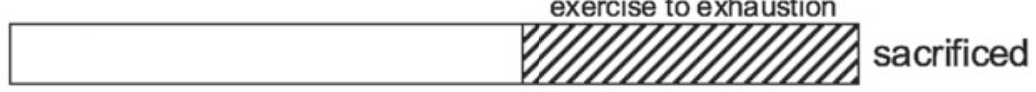

LEP

exercise preconditioning
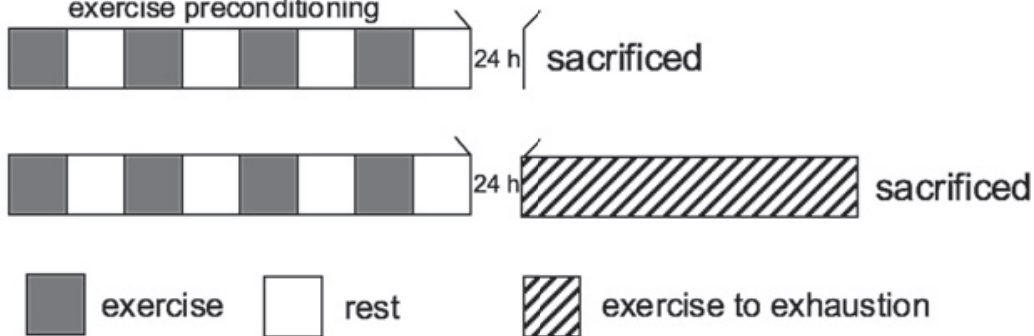

Figure 1. Exercise protocol. Rats in exercise groups ran on treadmill at speed of $30 \mathrm{~m} / \mathrm{minute}(0 \%$ degree $)$. C indicates control group; EE, exhaustive exercise group; LEP, late exercise preconditioning group; and LEP + EE, late exercise preconditioning plus exhaustive exercise group.

tective phase of EP via the analysis of LC3.

\section{Methods}

Animals: One hundred healthy adult (8-week-old) male Sprague-Dawley (SD) rats were recruited in this experiment. They were purchased from Animal Experimentation Center of Second Military Medical University and were housed in standard cages with 12-hour light/dark cycle and provided unlimited access to food and water. Animals were looked after by following the Guide for the Care and Use of Laboratory Animals, and experimental procedures were approved by the Ethics Committee for Science Research of the Shanghai University of Sport.

Experimental protocol: All animals were trained for treadmill habituation, which contained 5 consecutive days of treadmill exercise, $15 \mathrm{~m} /$ minute, $10-20$ minute/day. After the treadmill habituation, animals received 1 day of rest and were divided into four groups randomly comprising 1 control group and 3 exercise groups.

Control group (C): Rats were placed on nonmoving treadmill. The treadmill degree is 0 .

Exhaustive exercise group (EE): Rats run on treadmill until exhaustion. The treadmill speed is $30 \mathrm{~m} /$ minute.

Late exercise preconditioning group (LEP): Rats run on treadmill for EP model, which comprise 4 running time intervals that contain 10-minute running and rest.

Late exercise preconditioning plus exhaustive exercise group $(\mathrm{LEP}+\mathrm{EE})$ : Rats run on treadmill for $\mathrm{EP}$ model followed by exhaustive exercise after 24 hours.

All rats in the exercise groups received 5 minutes warm up and cool down training before or after the exercise. Rats in group LEP were sacrificed 24 hours after the exercise, the others were sacrificed 0.5 hours after exercise.

After exercise model, blood sample was drawn from the inferior caval vein. Ten rats were selected per group for perfusion fixation; thereafter, their hearts were excised and fixed by $4 \%$ paraformaldehyde for histology analysis.
The other rats' hearts were rapidly excised and stored at $-80^{\circ} \mathrm{C}$ (Figure 1).

Assessments of cardiac biomarkers: This was done using immunochemiluminescence to measure the serum cTnI levels, on Access 2 immunoassay system (Beckman Coulter, USA). This assay is based on a single step sandwich principle with paramagnetic particles coated as the solid phase and two monoclonal cTnI antibodies. The measurement range was $0.02 \sim 100 \mathrm{ng} / \mathrm{mL}$. The NTproBNP ELISA kit (R\&D Systems, USA) was used to measure serum NT-proBNP level. Double-antibody sandwich method was applied in this assay.

Chromotrope 2R-Brilliant green stain: Hearts were fixed by $4 \%$ paraformaldehyde for 24 hours and embedded with paraffin, sliced for $4 \mu \mathrm{m}$ sections, followed by deparaffinization and rehydration.

Chromotrope $2 \mathrm{R}$ has a strong affinity for ischemic/ hypoxic tissues and stains them red so that light green SF yellowish stain does not color them green; however, SF yellow stains the nonischemic/hypoxic tissues in green. The staining was performed to evaluate early myocardial ischemia/hypoxia. ${ }^{10)}$ Sections were stained in alum hematoxylin for 1 minute, followed by $5 \%$ Chromotrope $2 \mathrm{R}$ for 15 minutes, differentiated in $0.2 \%$ acetic acid thrice, stained in $5 \%$ light green SF yellowish for 15 minutes, and washed thrice with water. Eventually, the sections were covered with a coverslip and observed with a microscope (Olympus, Japan). In total, 25 images were captured per group for statistical analysis, which comprise 5 sections with 5 visual fields each.

In situ hybridization: For in situ hybridization, after blockage of endogenous peroxidase activity by $3 \%$ hydrogen peroxide, sections were digested with citric acid diluted pepsin, and then incubated with digoxin-labeled oligonucleotide probe hybridization solution, which contained three pieces of mRNA-probes of Kir6.2 and SUR2 A for each:

Sequence of Kir6.2 mRNA: 5'-AGGTA CCGTA CT CGG GAGAG GAGGG CCCGC TTCGT-3', 5'-TTCGC 
Table I. Alterations of Serum cTnI Levels in Rats

\begin{tabular}{lll}
\hline \multicolumn{1}{r}{ Group } & $n$ & $\mathrm{cTnI}(\mu \mathrm{g} / \mathrm{L})$ \\
\hline $\mathrm{C}$ & 20 & $0.02 \pm 0.01$ \\
$\mathrm{EE}$ & 18 & $3.87 \pm 5.04^{*}$ \\
$\mathrm{LEP}$ & 20 & $0.02 \pm 0.01$ \\
$\mathrm{LEP}+\mathrm{EE}$ & 19 & $0.83 \pm 0.63^{\#}$ \\
\hline
\end{tabular}

Values are the mean \pm SD. $C$ indicates control group; EE, exhaustive exercise group; LEP, late exercise preconditioning group; and LEP + $\mathrm{EE}$, late exercise preconditioning plus exhaustive exercise group. *Significantly different from group C, $P<0.05$; ${ }^{\#}$ Significantly different from group $\mathrm{EE}, P<0.05$.

CATGG TCTGG TGGCT CATCG CCCTT GCCCA-3', 5'-TGCAT CTTCA TGAAA ACGGC ACAGG CCCAT C GGCG-3';

Sequence of SUR2A mRNA: 5'-TTCTG TGGTA AC AAC ATCTC CTCCT ACAAC ATCTA-3', 5'-CAAAT TC ATC ACAAC ACATG GCTTC ATTTT CCTGG-3', 5'-C GGGT CCGAA GATAT GTTTT CTTCA TGAAT CCTC A-3'.

After hybridization treatment, the bound probe was detected using a streptavidin-biotin complex kit (Boster, China) with diaminobenzidine/peroxidase substrate to produce a brown-colored signal. Sections were then coverslipped and observed with microscope (Olympus, Japan). For negative controls, probe hybridization solution was replaced with PBS.

Real-time PCR: Total RNA was extracted from the heart cells using Trizol reagent according to the manufacturer's recommendations. The reverse transcription (RT) reaction was performed using SuperScript ${ }^{\mathrm{TM}}$ III Reverse Transcriptase (Invitrogen, USA). Primers for Kir6.2, SUR2A, and GAPDH were designed with Primer Express Software $\mathrm{v}$ 5.0 (AMI, USA) and demonstrated as following: Kir6.2: forward 5'-TATGTCCCTGCCCAACGAT-3', reverse 5'-A ACAGCAATAGGCTCCCAACT-3'; SUR2A: forward 5'CTACCGTCGCCCACCAGA-3', reverse 5'-CAACACCC ACGATGAACCTACA-3'; GAPDH: forward 5'-GGAAA GCTGTGGCGTGAT-3', reverse 5'-AAGGTGGAAGAAT GGGAGTT-3'. Real-time PCR was performed in the same wells of a 384-well plate in a real-time PCR detection system (ABI 7900, USA). Results were normalized by GAPDH and expressed as relative ratio.

Immunohistochemistry and Immunofluorescence: For immunohistochemistry, the sections were immersed in $3 \%$ hydrogen peroxide to block the endogenous peroxidase activity, and then immersed in sodium citrate buffer at $95^{\circ} \mathrm{C}$ for 10 minutes for antigen retrieval. Goat serum was applied to block nonspecific binding followed by overnight incubation of primary antibodies specific to BNP (BIOSS, China) at 1:200. A biotin conjugated second antibody complex kit (Boster, China) that contains streptavidin-biotin with a diaminoben-zidine/peroxidase was used to produce a brown color. Nuclei were stained using hematoxylin.

For immunofluorescence staining, after antigen retrieval, the sections were incubated with primary antibodies specific to Kir6.2 at 1:1000 (Alomone Labs, Israel) or SUR2A at 1:1000 (Santa Cruz Biotechnology, USA) overnight at $4^{\circ} \mathrm{C}$. Secondary antibody (Boster, China) was di-
Table II. Alterations of Serum NT-proBNP Levels in Rats

\begin{tabular}{lcc}
\hline \multicolumn{1}{c}{ Group } & $n$ & NT-proBNP $(\mu \mathrm{g} / \mathrm{L})$ \\
\hline $\mathrm{C}$ & 11 & $47.13 \pm 4.80$ \\
$\mathrm{EE}$ & 11 & $50.72 \pm 3.44^{*}$ \\
$\mathrm{LEP}$ & 11 & $42.21 \pm 3.65^{*}$ \\
$\mathrm{LEP}+\mathrm{EE}$ & 11 & $51.41 \pm 3.97$ \\
\hline
\end{tabular}

Values are the mean \pm SD. Abbreviations are the same as Table I.

*Significantly different from group $\mathrm{C}, P<0.05$.

luted at 1:1000 followed by detection using the SABCFITC or SABC-Cy3 (Boster, China) to produce florescence. The samples were then dried and coverslipped. Fluorescent images were collected with an inverted fluorescence microscope (Leica, Germany). To evaluate the autophagy in myocardium, primary antibody with stronger reactivity with the type II form of LC3B (Cell Signaling, USA) was used to reveal autophagosomes in cardiomyocytes. The sections were incubated with primary antibody (1:200) overnight $4{ }^{\circ} \mathrm{C}$. Alexa Fluor 488 conjugated second antibody (Abcam, UK) was applied at 1:500 for 1 hour at $37^{\circ} \mathrm{C}$. DAPI was used for nuclear staining. Laser scanning confocal, Zeiss LSM 700 (Carl Zeiss Jena, Germany), was used to capture images.

Western blotting: Myocardial proteins and samples were separated using standard SDS-PAGE techniques on a polyacrylamide gel, as described previously. Primary antibodies against Kir6.2 (Alomone Labs, Israel), SUR2A (Santa Cruz Biotechnology, USA), or LC3 (Novus Biologicals, USA) were diluted at 1:10,000, 1:3000, or 1: 1000, respectively. Horseradish peroxidase (HRP) conjugated secondary antibody (Cell Signaling, USA) was applied at 1:500 for 1 hour at room temperature. Results were normalized with $\beta$-actin or glyceraldehyde-3phosphate dehydrogenase (GAPDH) and expressed as the relative ratio.

Statistical analysis: The images were analyzed by ImagePro Plus 6.0 with positive events. Dates were indicated as mean $\pm \mathrm{SD}$, and the values were compared by one-way analysis of variance (SPSS19.0; SPSS, USA). When appropriately indicated by the presence of significant effects, group differences were determined via post hoc analysis. A value of $P<0.05$ was considered as statistically significant.

\section{Results}

Late cardioprotective effect of EP against exhaustive exercise: As Table I presents, serum cTnI levels in group EE were significantly higher than those in group $\mathrm{C}(P<$ $0.05)$. No significant differences were observed between group LEP and group $\mathrm{C}(P=\mathrm{NS})$. When compared with group EE, serum cTnI levels in group LEP + EE were significantly lower $(P<0.05)$. Table II presents that, compared with group $\mathrm{C}$, the serum NT-proBNP levels in group EE were significantly higher $(P<0.05)$; whereas, in those in group LEP were significantly lower $(P<0.05)$. No significant differences were observed between group $\mathrm{LEP}+\mathrm{EE}$ and group EE $(P=\mathrm{NS})$.

Images demonstrated that the nonhypoxic/ischemic tissue stained in light green color, whereas the hypoxic/ 
A
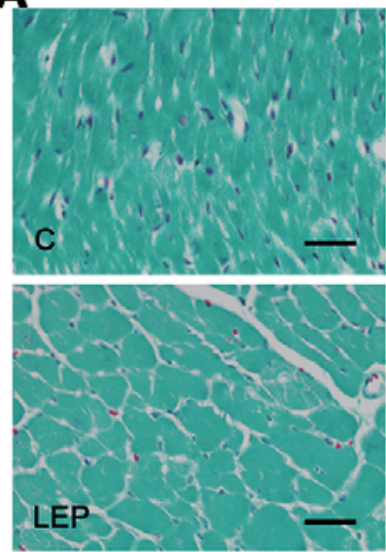

B

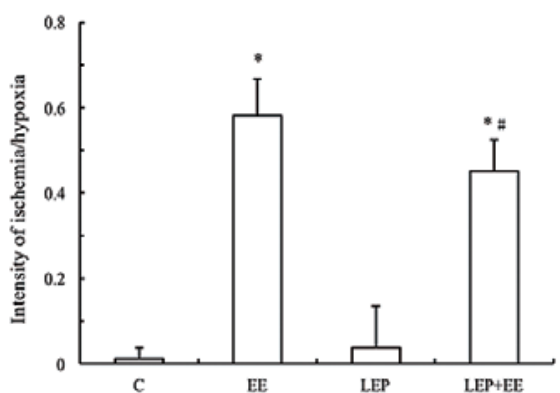

C
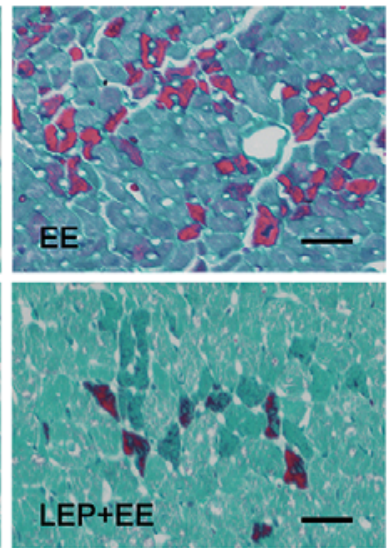

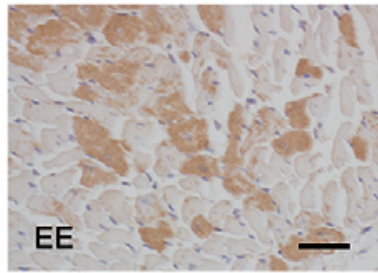

Ex

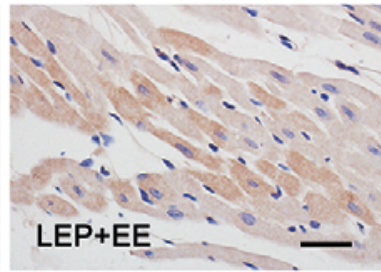

D

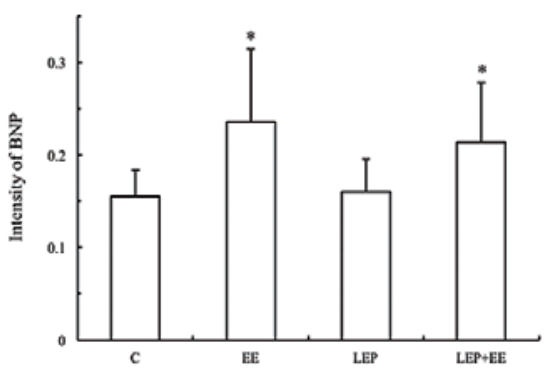

Figure 2. Late cardioprotective effect of EP against exhaustive exercise. A: Myocardium ischemia/hypoxia stained by Chromotrope 2R-Brillant green stain. Nonischemic/hypoxic tissue stained in light green color, whereas the ischemic/hypoxic cardiomyocytes stained in a vivid red color. Group EE revealed that ischemic/hypoxic cardiomyocytes stained in red color in contrast with those in group C and LEP, which displayed a bright green color. Group LEP + EE demonstrated some hypoxic and ischemic tissue scattered across the cardiomyocytes, bar $=20 \mu \mathrm{m}$. B: Quantitative analysis of ischemic/hypoxic cardiomyocytes $(n=25$ visuals/group). C: Immunohistochemistry staining of myocardial BNP. BNP immunoreactivity in myocardium were stained in brown color and diffused throughout the cytoplasm in cardiomyocytes, bar $=20 \mu \mathrm{m}$. D: Quantitative analysis of BNP immunoreactivity in cardiomyocytes $\left(n=25\right.$ visuals/group). Date are mean $\pm \mathrm{SD}$. ${ }^{*} P<0.05$ versus group C; $P<0.05$ versus group EE.

ischemic cardiomyocytes stained in red color (Figure 2A). Group EE revealed several ischemic/hypoxic cardiomyocytes stained in red color in contrast with those in group $\mathrm{C}$ and LEP, which displayed a bright green color. Group $\mathrm{LEP}+\mathrm{EE}$ demonstrated certain ischemic/hypoxic plaques scattered across the cardiomyocytes. Figure 2 revealed values of myocardial ischemia/hypoxia density. The myocardial ischemia/hypoxia density values in group EE significantly increased than those in group $\mathrm{C}$ (EE versus $\mathrm{C}$, $0.58 \pm 0.08$ versus $0.01 \pm 0.02, P<0.05)$; however, no differences were observed in group LEP (LEP versus C, $0.04 \pm 0.09$ versus $0.01 \pm 0.02, P=\mathrm{NS}$ ). Compared with group $\mathrm{EE}$, the myocardial ischemia/hypoxia density values in group LEP $+\mathrm{EE}$ were significantly lower $(\mathrm{LEP}+\mathrm{EE}$ versus EE, $0.45 \pm 0.07$ versus $0.58 \pm 0.08, P<0.05$ ).

BNP immunoreactivity in myocardium was stained in brown color and diffused throughout the cytoplasm in cardiomyocytes (Figure 2C). Compared with group $\mathrm{C}$, more cardiac cells demonstrated BNP immunoreactivity in group $\mathrm{EE}$ and $\mathrm{LEP}+\mathrm{EE}$. The intensity of BNP immunoreactivity in cardiomyocytes was evaluated and displayed as Figure 2D. Compared with group C, values of BNP immunoreactive density significantly increased in Group EE and Group LEP + EE (EE, LEP + EE versus
C, $0.24 \pm 0.08,0.21 \pm 0.06$ versus $0.16 \pm 0.03, P<0.05)$. No significant differences were found between Group $\mathrm{LEP}+\mathrm{EE}$ and group $\mathrm{EE}(\mathrm{LEP}+\mathrm{EE}$ versus $\mathrm{EE}, 0.21 \pm$ 0.06 versus $0.24 \pm 0.08, P=\mathrm{NS}$ ).

Expression of Kir6.2 in the late cardioprotective effects of EP: Kir6.2 mRNA was randomly distributed throughout the cytoplasm in the form of coarse granules (Figure 3 A). Kir6.2 mRNA levels in cardiomyocytes were assessed by RT-PCR. Compared with group C, no significant differences were found in group EE (EE versus C, $3.00 \pm$ 0.47 versus $3.55 \pm 1.04, P=\mathrm{NS}$ ) and group LEP (LEP versus $C, 3.18 \pm 0.53$ versus $3.55 \pm 1.04, P=\mathrm{NS})$. No significant differences were found between group LEP + $\mathrm{EE}$ and group $\mathrm{EE}(\mathrm{LEP}+\mathrm{EE}$ versus $\mathrm{EE}, 2.26 \pm 0.42$ versus $3.00 \pm 0.47, P=$ NS, Figure $3 \mathrm{~B}$ ).

The immunofluorescence staining of Kir6.2 revealed that Kir6.2 proteins were present in the cell membrane as well as in the striated pattern. It also demonstrated immunoreactivity along the sarcolemma, with bright green, diffuse punctate staining of the cardiomyocyte cytoplasm (Figure 3C). Myocardial Kir6.2 levels were detected using Western blotting as presented in Figure 3D. Kir6.2 levels in group EE significantly increased than those in group $\mathrm{C}$ (EE versus $\mathrm{C}, 0.56 \pm 0.07$ versus $0.28 \pm 0.07, P<0.05$ ). 
A
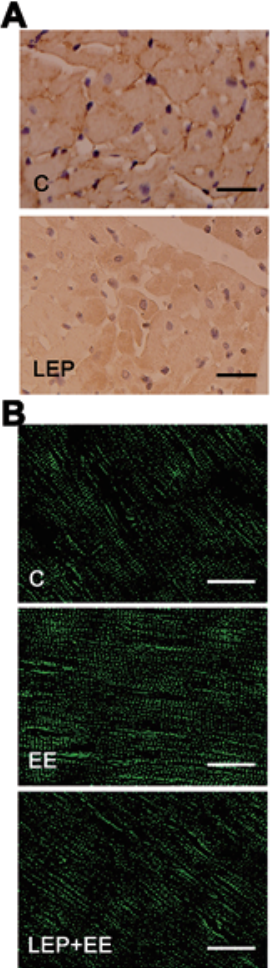

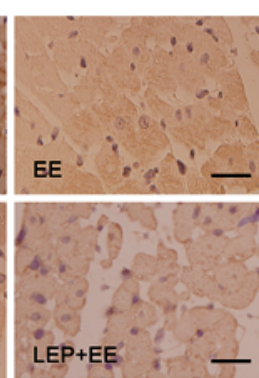

C

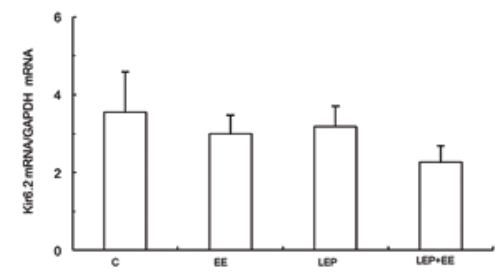

D
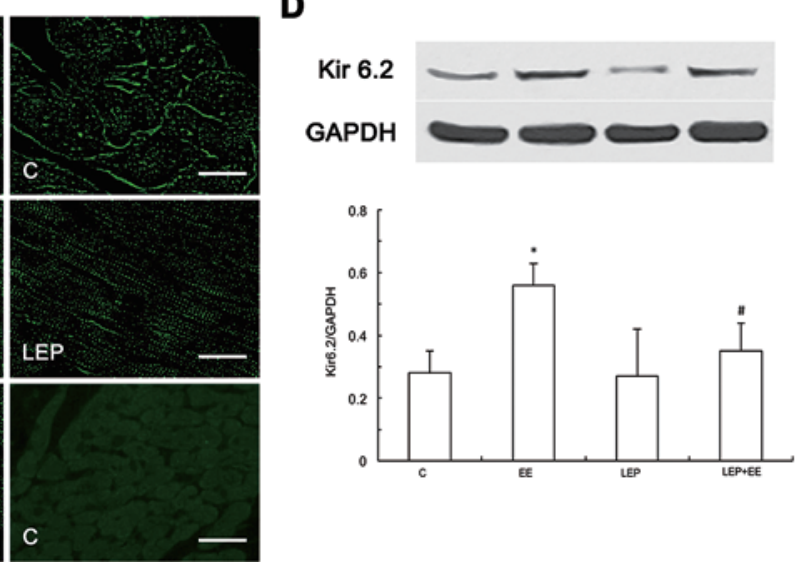

Figure 3. Alteration of Kir6.2 levels in the late cardioprotective effect of EP. A: In situ hybridization of Kir6.2 mRNA in cardiomyocytes. Kir6.2 mRNA demonstrated coarse granules randomly distributed in the cell cytoplasm in all groups. B: Myocardial Kir6.2 mRNA determined by real-time PCR ( $n=15$ rats/group). No significant differences were found in all groups. C: Immunofluorescence staining of myocardial Kir6.2. Kir6.2 protein demonstrated striated pattern in the cardiomyocytes, and along the sarcolemma, with bright green, diffuse punctate staining of the cardiomyocyte cytoplasm, bar $=20 \mu \mathrm{m}$. D: Kir6.2 levels in cardiomyocytes detected by Western blot analysis $(n=15$ rats/group). Date are mean \pm SD. $* P<0.05$ versus group $C ; \# P<0.05$ versus group EE.

No significant differences were observed between group LEP and group C (LEP versus C, $0.27 \pm 0.15$ versus 0.28 $\pm 0.07, P=\mathrm{NS})$. Kir6.2 levels in group LEP $+\mathrm{EE}$ were significantly lower than those in group $\mathrm{EE}(\mathrm{LEP}+\mathrm{EE}$ versus $\mathrm{EE}, 0.35 \pm 0.09$ versus $0.56 \pm 0.07, P<0.05$ ) .

Expression of SUR2A in the late cardioprotective effect of EP: The microscopic results of in situ hybridization are provided in Figure 4A. SUR2A mRNA demonstrated coarse granules randomly distributed in the cell cytoplasm. Real-time PCR results revealed that myocardial SUR2A mRNA levels in group EE were significantly higher than those in group $\mathrm{C}$ (EE versus $\mathrm{C}, 3.75 \pm 1.47$ versus $2.72 \pm 0.37, P<0.05$ ); however, no significant differences were observed in group LEP (LEP versus C, 2.78 \pm 0.40 versus $2.72 \pm 0.37, P=\mathrm{NS}$ ). No significant differences were detected between group LEP + EE and group $\mathrm{EE}(\mathrm{LEP}+\mathrm{EE}$ versus $\mathrm{EE}, 2.98 \pm 0.83$ versus $3.75 \pm 1.47$, $P=$ NS, Figure 4B).

The immunofluorescence staining of SUR2A revealed that SUR2A protein was present in the cell membrane as well as in the striated pattern. It also demonstrated immunoreactivity along the sarcolemma, with bright red, diffuse punctate staining of the cardiomyocyte cytoplasm (Figure 4C). Western blot analysis revealed that SUR2A levels in group EE were significantly higher than those in group C (EE versus $\mathrm{C}, 0.22 \pm 0.05$ versus $0.15 \pm 0.04, P$ $<0.05$ ); however, SUR2A levels in group LEP were significantly lower than in group $\mathrm{C}$ (LEP versus $\mathrm{C}, 0.10 \pm$ 0.08 versus $0.15 \pm 0.04, P<0.05)$. SUR2A levels in group LEP + EE were significantly lower than those in group $\mathrm{EE}(\mathrm{LEP}+\mathrm{EE}$ versus $\mathrm{EE}, 0.11 \pm 0.06$ versus 0.22 $\pm 0.05, P<0.05$, Figure 4D).

Expression of LC3 in the late cardioprotective effect of EP: LC3II demonstrated bright green puncta distributed in the cell cytoplasm in all groups. More cardiac cells demonstrated bright green puncta in group EE, LEP, and LEP $+\mathrm{EE}$ in comparison with group $\mathrm{C}$ (Figure 5A). Statistics of cardiomyocytes with LC3II puncta formation revealed that compared with group $\mathrm{C}$, the number of cardiac cells with bright green puncta in groups EE, LEP, and LEP + $\mathrm{EE}$ are significantly increased (EE, LEP, LEP + EE versus $\mathrm{C}, 0.26 \pm 0.08,0.11 \pm 0.03,0.12 \pm 0.03$ versus $0.07 \pm$ $0.02, P<0.05$, Figure 5B). When compared with group $\mathrm{EE}$, the number of cardiac cells with bright green puncta significantly decreased in group LEP $+\mathrm{EE}(\mathrm{LEP}+\mathrm{EE}$ versus EE, $0.12 \pm 0.03$ versus $0.26 \pm 0.08, P<0.05)$. LC 3II immunoreactive intensity values in group EE, LEP, and LEP + EE were significantly increased than those in 
A
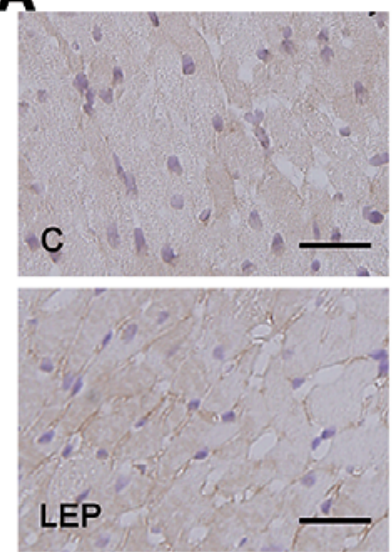

\section{B}
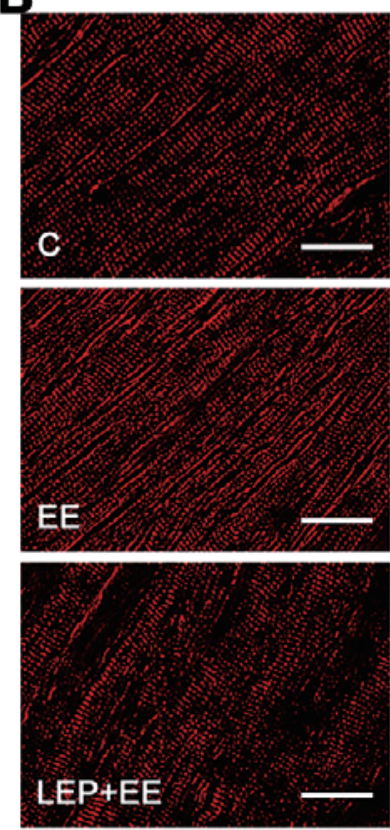
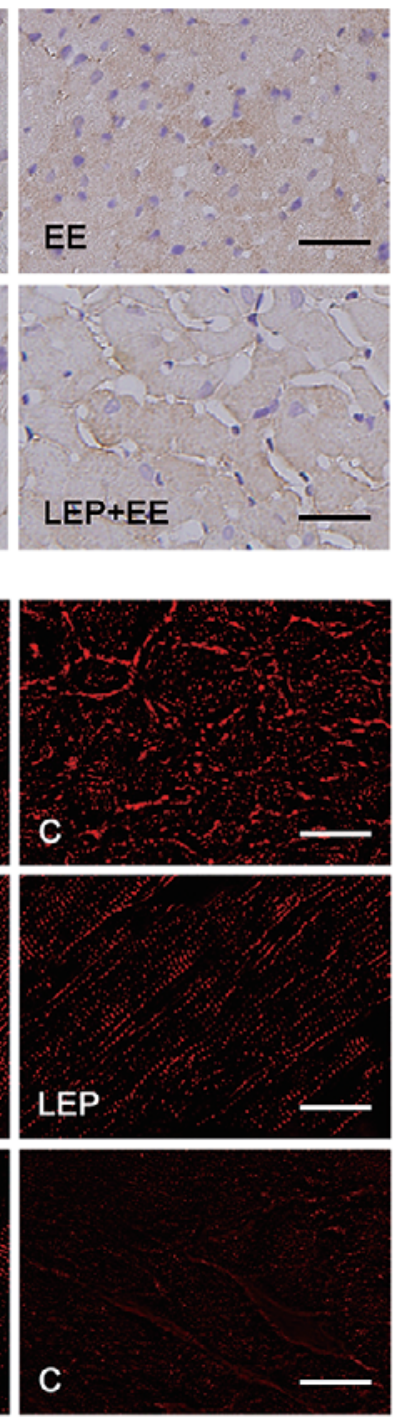

C

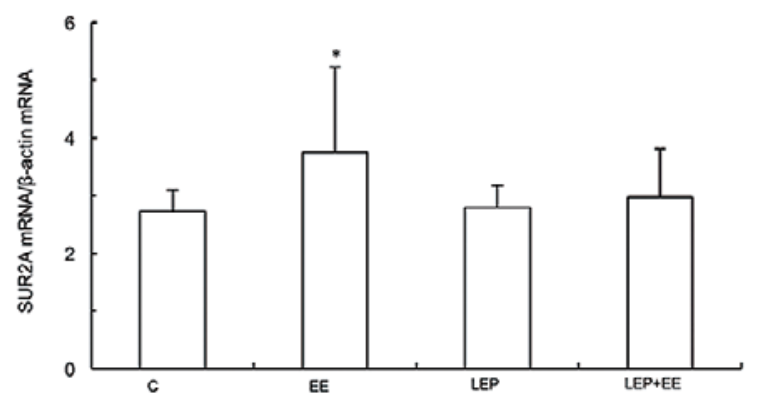

D
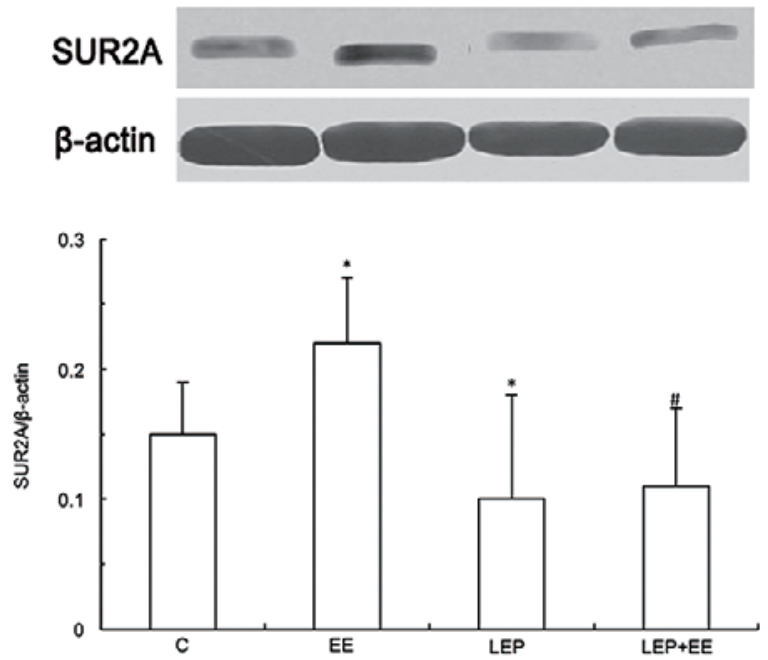

Figure 4. Alteration of SUR2A levels in the late cardioprotective effect of EP. A: In situ hybridization of SUR2A mRNA in cardiomyocytes. SUR2A mRNA demonstrated coarse granules randomly distributed in the cell cytoplasm in all groups bar $=20 \mu \mathrm{m}$. B: Myocardial SUR2A mRNA determined by real-time PCR ( $n=15$ rats/group). C: Immunofluorescence staining of myocardial SUR2A. SUR2A protein demonstrated striated pattern in the cardiomyocytes, and along the sarcolemma, with diffuse punctate staining of the cardiomyocyte cytoplasm, bar $=20 \mu \mathrm{m}$. D: SUR2A levels in cardiomyocytes detected by western blot analysis $(n=15$ rats/group). Date are mean $\pm \mathrm{SD}$. $* P<0.05$ versus group $C$; $\# P<0.05$ versus group EE.

group C (EE, LEP, LEP + EE versus C, $1.60 \pm 0.08,1.61$ $\pm 0.11,1.58 \pm 0.12$ versus $1.43 \pm 0.11, P<0.05$, Figure $5 C)$. Western bolt analysis showed the LC3IIlevels in groups EE, LEP, and LEP + EE were significantly increased than those in Group C (EE, LEP, LEP + EE versus $\mathrm{C}, 0.34 \pm 0.08,0.36 \pm 0.14,0.38 \pm 0.11$ versus 0.23 $\pm 0.08, P<0.05$, Figure 5D). The ratio of LC3II/LC3I in group $\mathrm{EE}$ was significantly higher than that in group $\mathrm{C}$ (EE versus C, $2.02 \pm 0.76$ versus $1.54 \pm 0.32, P<0.05$ ). When compared with group EE, the ratio of LC3II/LC3I in group LEP + EE was significantly lower $(\mathrm{LEP}+\mathrm{EE}$ versus EE, $1.63 \pm 0.34$ versus $2.02 \pm 0.76, P<0.05$, Figure $5 \mathrm{E})$.

\section{Discussion}

Cardiac troponin I (cTnI), a sensitive biomarker of myocardial injury, is often used to diagnose myocardial infarction in clinic. ${ }^{11)}$ In some cases, cTnI acts as a monitor to evaluate the prolonged exercise induced minor myocardial injury. ${ }^{12)}$ BNP is a biomarker for cardiac dysfunction. ${ }^{13)}$ It is secreted by the heart in response to excessive stretching of the heart muscle cells and is released as preproBNP, ${ }^{14)}$ which comprises the biologically active BNP and the inactive metabolite N-proBNP. Researchers have reported that serum NT-proBNP and cTnI increased after prolonged endurance exercise, which is in correspondence with the decline of cardiac contractility; ${ }^{12,15)}$ how- 

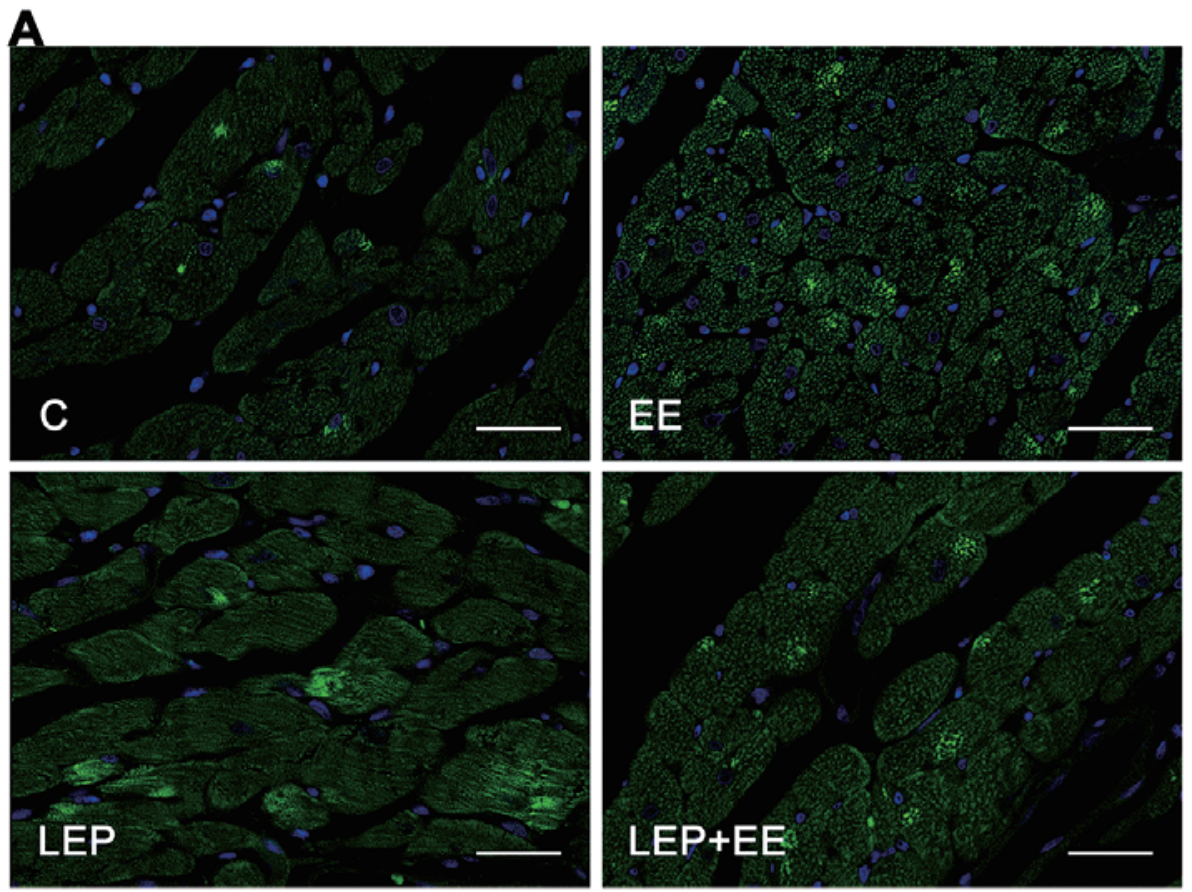

\section{B}

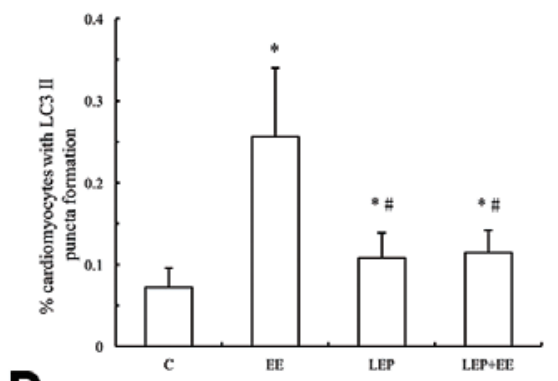

D

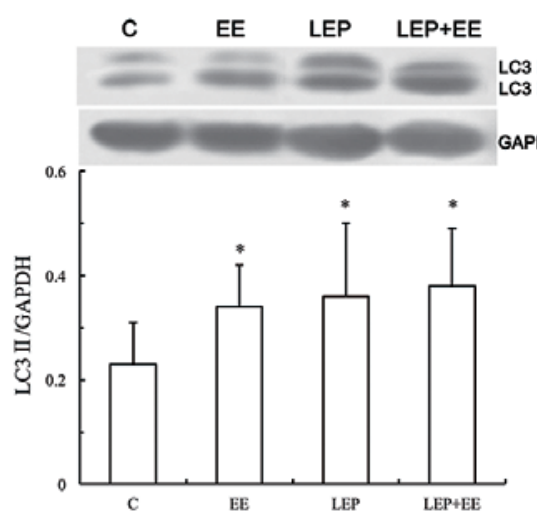

C

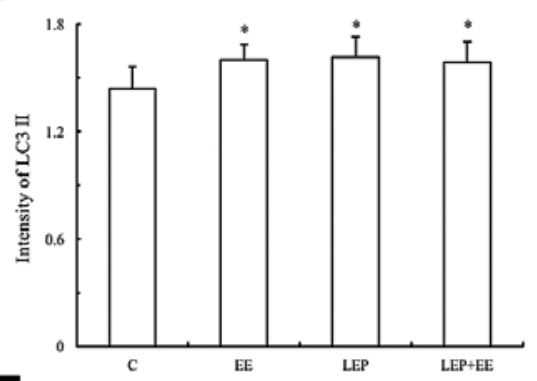

$\mathbf{E}$

\section{LC3।}

APDH

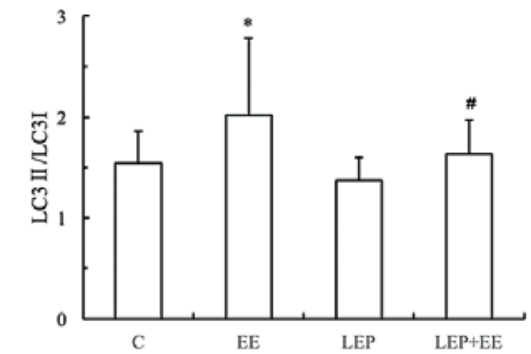

Figure 5. Alteration of LC3 in the late cardioprotective effect of E P. A: Immunofluorescence staining of myocardial LC3 II. LC3 II demonstrated bright green puncta randomly distributed in cell cytoplasm in all groups, bar $=20 \mu \mathrm{m}$. B: Quantitative analysis of cardiomyocytes with LC3 II puncta ( $n=25$ visuals/group). C: Quantitative analysis of LC3 II immunoreactivity in myocardium ( $n=25$ visuals/group). D: LC3 II levels in myocardium by western blot analysis ( $n=15$ rats/group). E: Ratio of LC3 II/LC3 I in myocardium ( $n=15$ rats/group). Date are mean $\pm \mathrm{SD} .{ }^{*} P<0.05$ versus group $\mathrm{C} ;{ }^{*} P<0.05$ versus group EE.

ever, the detection of BNP in heart during exercise is hardly reported. In our study, in addition to the high levels of serum cTnI and NT-proBNP, increased ischemic/hy- poxic cardiomyocytes and over-expressed BNP were observed after exhaustive exercise, suggesting that exhaustive exercise might cause myocardial damage and cardiac 
wall stress or dysfunction.

EP exerts cardioprotective effects and comprises two distinct phases: an early phase and a late phase. The late cardioprotection of EP has been observed by other researchers in myocardial infarction animal models. In a study by Domenech, et al., ${ }^{16,17)}$ dogs ran on a treadmill for 5 periods of 5 minutes each at $6 \mathrm{~km} /$ hour along with 5 minute periods of rest as a preconditioning protocol. Results revealed that infarct size induced by $I / R$ injury in $E P$ group significantly decreased in the late preconditioned phase, but the decline in the cardiac function was not significantly improved. Similarly, in this experiment, the cardiac BNP along with serum NT-proBNP remain at high levels after exhaustive exercise with EP, except the reduction of serum cTnI level and cardiac hypoxia/ischemia. These results suggest that EP might contribute to the reduction of myocardial injury and ischemia/hypoxia; however, cardiomyocytes are still under over-stretched during exhaustive exercise, which might promote cardiac BNP and serum NT-proBNP. Importantly, evidences revealed that the late cardioprotection of EP against I/R injury was abolished by $\mathrm{K}_{\text {ATP }}$ channel inhibitor, ${ }^{2,6)}$ indicating that cardiac $K_{\text {ATP }}$ channels are involved in the cardioprotection of EP.

It is widely assumed that $\mathrm{K}_{\text {ATP }}$ channels are abundantly expressed in the cardiacmyocytes. Cardiac $\mathrm{K}_{\text {ATP }}$ channels comprise pore-forming Kir6.2 and regulatory SUR2A subunits. ${ }^{18)}$ Kir6.2 subunits form a pore-forming structure through which $\mathrm{K}^{+}$ions transverse the membrane and SUR2A subunits assemble with $\mathrm{K}^{+}$ions to modulate the channel's function and to confer unique pharmacological properties on the channel complex. ${ }^{19)}$ Our study is a comprehensive analysis of the localization of Kir6.2 and SUR2A in cardiomyocytes. Both of Kir6.2 mRNA and SUR2A mRNA demonstrated coarse granules randomly distributed in the cell cytoplasm. Similar results revealed Kir6.2 mRNA to be expressed in the left and right ventricles in hypoxic preconditioning of rabbit hearts $;^{20)}$ however, the localization of SUR2A mRNA in the cardiomyocytes has rarely been reported. The present study may be the first to measure the expression of SUR2A mRNA using in situ hybridization. Moreover, both Kir6.2 and SUR2A expressed striated patterns in the cardiomyocytes and along the sarcolemma, with diffuse punctate staining of the cardiomyocyte cytoplasm. Nevertheless, Li, et al. ${ }^{21)}$ found Kir6.2 to be localized primarily to the transverse tubule network of isolated adult mouse cardiomyocytes. Immunofluorescence demonstrated that SUR2A presented a striated pattern in the cell membrane and a punctate pattern in the cytoplasm of cardiomyocytes. Immunoelectron micrography further revealed SUR2A to be expressed in the mitochondria, as well as in the cell membrane. Some immunoreactivity was localized in t-tubules near the I bands of cardiac muscle fibers. ${ }^{22)}$ In addition, immunocytochemistry of isolated ventricular myocytes revealed colocalization of Kir6.2 and SUR2 in a striated sarcomeric pattern, suggesting t-tubular expression of both proteins. $^{23)}$

$\mathrm{K}_{\text {ATP }}$ channel subunits mRNA levels in the myocardial cells were reported to be changed by hypoxia, ischemia, and exercise. Crawford, et al. ${ }^{24)}$ reported that chronic mild hypoxia protects heart-derived $\mathrm{H} 9 \mathrm{c} 2$ cells against acute hypoxia/reoxygenation by regulating expression of the $\mathrm{K}_{\text {ATP }}$ channel subunits. SUR2A mRNA levels in the cells exposed to $\mathrm{PO}_{2}=100 \mathrm{mmHg}$ was $>$ 2-fold higher than those exposed to $\mathrm{PO}_{2}=144 \mathrm{mmHg}$. In contrast, Kir6.2 mRNA levels did not change under mild hypoxic conditions. Melamed-Frank, et $a l .{ }^{25)}$ reported that Kir6.2 mRNA levels significantly decreased by hypoxia in vivo. In Zingman, et al.'s ${ }^{26)}$ study, mice were exercised daily for 5 consecutive days at a speed of $12 \mathrm{~m} /$ minute and inclination of $15^{\circ}$ for 45 minutes. In this way, SUR2A mRNA levels were significantly increased, but Kir6.2 mRNA levels did not change significantly after exercise. Thus, SUR2A may be a limiting factor in the determination of the number of $\mathrm{K}_{\text {ATP }}$ channels, since SUR2A mRNA levels were upregulated by metabolic stress of the myocardium. ${ }^{27)}$ In the present study, Kir6.2 mRNA levels did not significantly change in groups subjected to exercise preconditioning with exhaustive exercise-induced myocardial injury; however, the upregulation of SUR2A mRNA levels was found to be associated with exhaustive exercise. The exhaustive exercise caused acute myocardial injury in rats, with myocardial cell ischemia, hypoxia, and energy depletion. Upregulation of SUR2A transcription levels may play a decisive role in the increase in the number of $\mathrm{K}_{\text {ATP }}$ channels. This may create a cardiac phenotype with increased resistance to hypoxia, ischemia, or even myocardial infarction. The underlying mechanisms by which levels of Kir6.2 mRNA and SUR2A mRNA differ in the cardioprotective phenotype produced by exercise preconditioning merit further exploration, as do the changes in protein levels.

It has been recognized that levels of Kir6.2 and SUR2A increase in ischemic/hypoxia/exercise-induced cardioprotection scenarios. $\mathrm{K}_{\mathrm{ATP}}$ channel subunits were upregulated ischemic/hypoxic conditions in both in vitro and in vivo experiments. ${ }^{24,27)}$ Reports demonstrated that 12 weeks of exercise can reduce myocardial infarct size after $\mathrm{I} / \mathrm{R}$ injury in rat hearts. ${ }^{28)}$ This cardioprotective phenotype is characterized by an increased expression of both of Kir 6.2 and SUR2A subunit; however, upregulation of Kir6.2 and SUR2A subunits in ischemic/hypoxic cardiomyocytes might contribute to the substantial time-dependent increases in effective refractory period-heterogeneity during ventricular fibrillation, and the blockage of $\mathrm{K}_{\text {ATP }}$ channels could inhibit ventricular arrhythmias induced by ischemia/ reperfusion injury. ${ }^{29,30)}$ Our study suggests that exhaustive exercise can cause acute myocardial injury, ischemia/hypoxia of myocardial cells, cardiac stress or dysfunction, and so increase Kir6.2 and SUR2A protein levels and regulate the number of $\mathrm{K}_{\text {ATP }}$ channels. Opening of cardiac $\mathrm{K}_{\text {ATP }}$ channels exerts cardioprotective phenotype; ${ }^{31)}$ however, over-expression of $\mathrm{K}_{\text {ATP }}$ channels subunits might increase the risk of ventricular arrhythmias. Interestingly, implementation of EP before exhaustive exercise significantly decreased Kir6.2 and SUR2A protein expression, suggesting that EP-induced late cardioprotection against exhaustive exercise in rats through decreased levels of $\mathrm{K}_{\text {ATP }}$ channel subunit Kir6.2 and SUR2A protein. Eventually, the influence of EP upon the level of translation of SUR2A was found to be more significant than that of 
with Kir6.2. The cardiac $\mathrm{K}_{\text {ATP }}$ channel protein complex may contain other enzymes. ${ }^{32)}$ Of these, SUR2A is the least expressed protein, making it a limiting factor in the determination of the number of cardiac $\mathrm{K}_{\text {ATP }}$ channels. ${ }^{23)}$ In the present study, both the transcription and translation levels of SUR2A changed more significantly than those of Kir6.2, indicating that SUR2A may play a decisive role in the expression of cardiac $\mathrm{K}_{\mathrm{ATP}}$ channels and EP impact mainly on SUR2A expression.

LC3 protein is associated with the maturation progress of autophagosome. It contains two forms: LC3 I located in cytosolic fractions, and LC3 II located both to inner and outer of the autophagy membrane. Once autophagy is activated, LC3 II is recruited in autophagy membrane via conversion of LC3 I to LC3 II, which is regarded as "carrier flux" to accelerate the formation of autophagosomes. ${ }^{33)}$ Hence, it is widely served as key molecule to monitor autophagosomes and autophagic activity. ${ }^{34)}$ Researches found that strenuous exercise improved formation of autophagosomes in cardiomyocytes and elevated myocardial LC3 II levels, which might contribute to the beneficial metabolic effect of exercise and clearance of strenuous exercise-induced overproduced reactive oxygen species (ROS) ${ }^{8,9)}$ In our experiment, the upregulation of autophagosomes and LC3 II levels were observed in all exercise groups, implying that exhaustive and high intensive interval exercise could cause promotion of autophagy in cardiomyocytes. To our surprise, accumulation of autophagosomes and LC3 II turnover in group $\mathrm{EE}$ are much more upregulated than other exercise groups, suggesting that excessive autophagy might occur during exhaustive exercise. Combining the previous evidences that exhaustive exercise could cause myocardial damage and cardiac wall stress, we proposed that excessive autophagy in cardiomyocytes might contribute to the damage of cardiomyoctyes. Since autophagy might have side effects in response to mediate stress-induced adaptation and damage control in cells. ${ }^{35)}$ Reach demonstrated that excessive autophagy might aggravate $\mathrm{I} / \mathrm{R}$ induced myocardial injury both in vitro and vivo, ${ }^{36,37)}$ whereas swimming training significantly reduced the myocardial infarct size and abolished the induction of autophagy in mice with acute myocardial infarction. ${ }^{38)}$ Our results demonstrated that EP reversed exhaustive exercise-induced autophagy by reducing the autophagosomes and LC3 II turnover in cardiomyocytes, and attenuated myocardial injury. This is in accordance with the previous study that exercise training could attenuate doxorubicin-induced autophagy and toxicity in cardiomyocytes. Collectively, our research indicates that EP brings out late cardioprotective effects against exhaustive exercise-induced myocardial injury through the reduction of autophagic markers in cardiomyocytes.

\section{Conclusion}

The present work demonstrated that EP exerts cardioprotective effects through the reduction of exhaustive exercise-induced myocardial injury; however, could not significantly improve the cardiac stress or dysfunction. Kir 6.2 and SUR2A expressed striated patterns in the cardiomyocytes and along the sarcolemma, with diffuse punctate staining of the cardiomyocyte cytoplasm. Both the transcription and translation levels of Kir6.2 and SUR2A after exhaustive exercise were crippled by EP in late cardioprotective phase, as well as exhaustive exercise-increased autophagosomes and LC3 II turnover in cardiomyocytes. Therefore, theses results suggest that the downregulation of cardiac $\mathrm{K}_{\text {ATP }}$ channels and autophagy are involved in EP-induced late cardioprotection against exhaustive exercise in rats.

\section{Disclosures}

Conflicts of interest: The authors have no conflicts of interest to disclose.

\section{References}

1. Hao Z, Pan SS, Shen YJ, Ge J. Exercise preconditioninginduced early and late phase of cardioprotection is associated with protein kinase C epsilon translocation. Circ J 2014; 78: 1636-45.

2. Parra VM, Macho P, Domenech RJ. Late cardiac preconditioning by exercise in dogs is mediated by mitochondrial potassium channels. J Cardiovasc Pharmacol 2010; 56: 268-74.

3. McGinnis GR, Ballmann C, Peters B, et al. Interleukin-6 mediates exercise preconditioning against myocardial ischemia reperfusion injury. Am J Physiol Heart Circ Physiol 2015; 308: H1423-33.

4. Marongiu E, Crisafulli A. Cardioprotection acquired through exercise: the role of ischemic preconditioning. Curr Cardiol Rev 2014; 10: 336-48.

5. Shen YJ, Pan SS, Zhuang T, Wang FJ. Exercise preconditioning initiates late cardioprotection against isoproterenol-induced myocardial injury in rats independent of protein kinase C. J Physiol Sci 2011; 61: 13-21.

6. Chicco AJ, Johnson MS, Armstrong CJ, et al. Sex-specific and exercise-acquired cardioprotection is abolished by sarcolemmal KATP channel blockade in the rat heart. Am J Physiol Heart Circ Physiol 2007; 292: H2432-7.

7. Brown DA, Lynch JM, Armstrong CJ, et al. Susceptibility of the heart to ischaemia-reperfusion injury and exercise-induced cardioprotection are sex-dependent in the rat. J Physiol 2005; 564: 619-30.

8. He C, Bassik MC, Moresi V, et al. Exercise-induced BCL2regulated autophagy is required for muscle glucose homeostasis. Nature 2012; 481: 511-5.

9. Li H, Miao W, Ma J, et al. Acute Exercise-Induced Mitochondrial Stress Triggers an Inflammatory Response in the Myocardium via NLRP3 Inflammasome Activation with Mitophagy. Oxid Med Cell Longev 2016; 2016: 1987149.

10. Bi HT, Yang Y, Liu X, et al. Application and comparison of three special stains in the postmortem diagnosis of acute myocardial infarction. Chin J Forensic Med 2013; 28: 330-2.

11. Kim M, Son M, Lee DH, Park K, Park TH. Troponin-I Level After Major Noncardiac Surgery and Its Association With LongTerm Mortality. Int Heart J 2016; 57: 278-84.

12. La Gerche A, Burns AT, Mooney DJ, et al. Exercise-induced right ventricular dysfunction and structural remodelling in endurance athletes. Eur Heart J 2012; 33: 998-1006.

13. Kaneko H, Neuss M, Weissenborn J, Butter C. Role of Right Ventricular Dysfunction and Diabetes Mellitus in N-terminal pro-B-type Natriuretic Peptide Response of Patients With Severe Mitral Regurgitation and Heart Failure After MitraClip. Int Heart J 2017; 58: 225-31.

14. Takeishi Y. Biomarkers in heart failure. Int Heart J 2014; 55: 474-81. 
15. Ljones K, Ness HO, Solvang-Garten K, Gaustad SE, Andre Hoydal M. Acute exhaustive aerobic exercise training impair cardiomyocyte function and calcium handling in SpragueDawley rats. PLoS One 2017; 12: e0173449.

16. Parra VM, Macho P, Sanchez G, Donoso P, Domenech RJ. Exercise preconditioning of myocardial infarct size in dogs is triggered by calcium. J Cardiovasc Pharmacol 2015; 65: 276-81.

17. Domenech R, Macho P, Schwarze H, Sanchez G. Exercise induces early and late myocardial preconditioning in dogs. Cardiovasc Res 2002; 55: 561-6.

18. Inagaki N, Gonoi T, Clement JP, et al. A family of sulfonylurea receptors determines the pharmacological properties of ATPsensitive K+ channels. Neuron 1996; 16: 1011-7.

19. Nakaya H. Role of ATP-sensitive $\mathrm{K}+$ channels in cardiac arrhythmias. J Cardiovasc Pharmacol Ther 2014; 19: 237-43.

20. Choi SW, Ahn JS, Kim HK, et al. Increased Expression of ATPsensitive K Channels Improves the Right Ventricular Tolerance to Hypoxia in Rabbit Hearts. Korean J Physiol Pharmacol 2011; 15: 189-94.

21. Li J, Kline CF, Hund TJ, Anderson ME, Mohler PJ. Ankyrin-B regulates Kir6.2 membrane expression and function in heart. J Biol Chem 2010; 285: 28723-30.

22. Zhou M, He HJ, Suzuki R, et al. Localization of sulfonylurea receptor subunits, SUR2A and SUR2B, in rat heart. J Histochem Cytochem 2007; 55: 795-804.

23. Morrissey A, Rosner E, Lanning J, et al. Immunolocalization of KATP channel subunits in mouse and rat cardiac myocytes and the coronary vasculature. BMC Physiol 2005; 5: 1 .

24. Crawford RM, Jovanovic S, Budas GR, et al. Chronic mild hypoxia protects heart-derived H9c2 cells against acute hypoxia/ reoxygenation by regulating expression of the SUR2A subunit of the ATP-sensitive K+ channel. J Biol Chem 2003; 278 : 31444-55.

25. Melamed-Frank M, Terzic A, Carrasco AJ, Nevo E, Avivi A, Levy AP. Reciprocal regulation of expression of pore-forming KATP channel genes by hypoxia. Mol Cell Biochem 2001; 225: 145-50.

26. Zingman LV, Zhu Z, Sierra A, et al. Exercise-induced expression of cardiac ATP-sensitive potassium channels promotes action potential shortening and energy conservation. J Mol Cell Cardiol 2011; 51: 72-81.

27. Du Q, Jovanovic S, Clelland A, et al. Overexpression of SUR2A generates a cardiac phenotype resistant to ischemia. Faseb J 2006; 20: 1131-41.

28. Brown DA, Chicco AJ, Jew KN, et al. Cardioprotection afforded by chronic exercise is mediated by the sarcolemmal, and not the mitochondrial, isoform of the KATP channel in the rat. J Physiol 2005; 569: 913-24.

29. Vajda S, Baczko I, Lepran I. Selective cardiac plasma-membrane $\mathrm{K}(\mathrm{ATP})$ channel inhibition is defibrillatory and improves survival during acute myocardial ischemia and reperfusion. Eur $\mathrm{J}$ Pharmacol 2007; 577: 115-23.

30. Farid TA, Nair K, Masse S, et al. Role of KATP channels in the maintenance of ventricular fibrillation in cardiomyopathic human hearts. Circ Res 2011; 109: 1309-18.

31. Niwano S, Hirasawa S, Niwano H, et al. Cardioprotective effects of sarcolemmal and mitochondrial K-ATP channel openers in an experimental model of autoimmune myocarditis. Role of the reduction in calcium overload during acute heart failure. Int Heart J 2012; 53: 139-45.

32. Hund TJ, Mohler PJ. Differential roles for SUR subunits in KATP channel membrane targeting and regulation. Am J Physiol Heart Circ Physiol 2011; 300: H33-5.

33. Klionsky DJ, Abdelmohsen K, Abe A, et al. Guidelines for the use and interpretation of assays for monitoring autophagy (3rd edition). Autophagy 2016; 12: 1-222.

34. Slobodkin MR, Elazar Z. The Atg8 family: multifunctional ubiquitin-like key regulators of autophagy. Essays Biochem 2013; 55: 51-64.

35. Chiong M, Wang ZV, Pedrozo Z, et al. Cardiomyocyte death: mechanisms and translational implications. Cell Death Dis 2011; 2: e244.

36. Valentim L, Laurence KM, Townsend PA, et al. Urocortin inhibits Beclin1-mediated autophagic cell death in cardiac myocytes exposed to ischaemia/reperfusion injury. J Mol Cell Cardiol 2006; 40: 846-52.

37. Zeng M, Wei X, Wu Z, et al. NF-kappaB-mediated induction of autophagy in cardiac ischemia/reperfusion injury. Biochem Biophys Res Commun 2013; 436: 180-5.

38. Tao L, Bei Y, Lin S, et al. Exercise Training Protects Against Acute Myocardial Infarction via Improving Myocardial Energy Metabolism and Mitochondrial Biogenesis. Cell Physiol Biochem 2015; 37: 162-75. 